\title{
The wavelet transform on Sobolev spaces and its approximation properties
}

\author{
Andreas Rieder* \\ Universität des Saarlandes, Fachbereich Mathematik, Bau 38, \\ W-6600 Saarbrücken, Federal Republic of Germany
}

Received December 28, 1989/June 16, 1990

Summary. We extend the continuous wavelet transform to Sobolev spaces $H^{s}(\mathbb{R})$ for arbitrary real $s$ and show that the transformed distribution lies in the fiber spaces $L_{2}\left(\left(\mathbb{R}_{0}, \frac{d a}{a^{2}}\right), H^{s}(\mathbb{R})\right) \cong H^{0, s}\left(\mathbb{R}^{2}, \frac{d a d b}{a^{2}}\right)$. This generalisation of the wavelet transform naturally leads to a unitary operator between these spaces.

Further the asymptotic behaviour of the transforms of $L_{2}$-functions for small scaling parameters is examined. In special cases the wavelet transform converges to a generalized derivative of its argument. We also discuss the consequences for the discrete wavelet transform arising from this property. Numerical examples illustrate the main result.

Subject classifications: AMS (MOS): 44 A15, 65D99

\section{Introduction}

The wavelet transform (WT) is a tool for analyzing and synthesizing signals with many applications in geophysics [5], acoustics [6], and quantum theory [12]. It has a lot of advantages compared to the Fourier transform, e.g. the high frequency components are studied with sharper time resolution than low frequency components [2].

The transformed signal is composed by its inner product with shifted and scaled versions of a fixed function called analyzing or basic wavelet.

Let $f \in L_{2}(\mathbb{R})$ be the signal and $\psi \in L_{2}(\mathbb{R})$ the analyzing wavelet. The mapping

$$
f(\cdot) \mapsto|a|^{-1 / 2}\left\langle f, \psi\left(\frac{\cdot-b}{a}\right)\right\rangle_{0}, \quad b \in \mathbb{R}, \quad a \in \mathbb{R} \backslash\{0\},
$$

\footnotetext{
* Supported by the Deutsche Forschungsgemeinschaft under grant Lo 310/2-4 
describes the analysis of $f$ (up to constant factor), where $\langle\cdot, \cdot\rangle_{0}$ denotes the inner product in $L_{2}(\mathbb{R})$. With an admissibility condition on $\psi$ the right hand side of (1) is an element in $L_{2}\left((\mathbb{R} \times \mathbb{R} \backslash\{0\}), \frac{d b d a}{a^{2}}\right)$ and it is possible to synthesize $f$ by these moments.

In the literature one often finds the definition of the wavelet transform via an irreducible unitary representation of the group of affine-linear transformations of the real axis ('ax $+b$ '-group). Hence its essential properties are abstractly proved by help of group theory (orthogonality relations).

For a detailed description of these group-theoretical aspects we refer to Grossmann, Morlet, and Paul [7,8].

In the next section some known results will be verified without group-theoretical arguments in such a way that the extension of the wavelet transform to Sobolev spaces becomes obvious. It will be seen that the signal and the wavelet transform share the same Sobolev order. The discrete wavelet transform was already extended to the spaces $H^{s}(\mathbb{R})$ by Daubechies [2] via the concept of frames but only for very special choices of basic wavelets.

The preponderant part of the paper deals with the asymptotic behaviour of (1) for small $a$. Without a heuristic frequency analysis our inquiry explains the basics for the widespread use of wavelet techniques in edge detection and pattern recognition. It turns out that the right hand side of (1) converges to a derivative of $f$, as already observed for a very special example in [8], p. 306, for a great class of basic wavelets $\psi$ (in particular for all compactly supported). In Sect. 5 we apply the results to show the approximation properties of the discrete wavelet transform. Again derivatives of the transformed signal are computed. Numerical tests in Sect. 6 verify the theoretical results.

\section{The wavelet transform}

We define with the help of the shift-operator

$$
\left(T^{b} g\right)(x)=g(x-b), \quad b \in \mathbb{R}
$$

and the dilation-operator

$$
\left(D^{a} g\right)(x)=|a|^{-1 / 2} g\left(\frac{x}{a}\right), \quad a \in \mathbb{R}_{0}:=\mathbb{R} \backslash\{0\}
$$

a unitary transformation $U(b, a): L_{2}(\mathbb{R}, d t) \rightarrow L_{2}(\mathbb{R}, d t)$, where $d t$ denotes the Lebesgue-measure, by

$$
(U(b, a) g)(x)=\left(T^{b} D^{a} g\right)(x)=|a|^{-1 / 2} g\left(\frac{x-b}{a}\right), \quad(b, a) \in \mathbb{R} \times \mathbb{R}_{0} .
$$

To simplify further calculations we introduce the Fourier transform

$$
(F f)(\omega)=\hat{f}(\omega)=\frac{1}{\sqrt{2 \pi}} \int_{\mathbb{R}} f(x) \mathrm{e}^{-\mathrm{i} x \omega} d x, \quad \omega \in \mathbb{R},
$$


leading to

$$
\begin{aligned}
& F T^{b}=\mathrm{e}^{-\mathrm{i} b(\cdot)} F \\
& F D^{a}=D^{1 / a} F .
\end{aligned}
$$

Hence we get

$$
(U(b, a) g)^{\wedge}(\omega)=F\left(T^{b} D^{a} g\right)(\omega)=\mathrm{e}^{-\mathrm{i} b \omega}|a|^{1 / 2} \hat{g}(a \omega)
$$

In the sequel we describe the wavelet transform, based on a function $\psi$.

Definition 2.1. A function $\psi \in L_{2}(\mathbb{R}, d t)$ is admissible if and only if $\psi$ is not identical to zero and $\langle U(\cdot, \cdot) \psi, \psi\rangle_{0}$ lies in $L_{2}\left(\mathbb{R} \times \mathbb{R}_{0}, \frac{d b d a}{a^{2}}\right)$, i.e.

$$
\int_{\left.\mathbb{R}_{0}\right)} \int_{\mathbb{R}}\left|\langle U(b, a) \psi, \psi\rangle_{0}\right|^{2} \frac{d b d a}{a^{2}}<\infty .
$$

With '*' denoting the convolution we reformulate the admissibility condition (8) as

$$
\begin{aligned}
\int_{\mathbb{R}_{0}} \int_{\mathbb{R}}\left|\left\langle T^{b} D^{a} \psi, \psi\right\rangle_{0}\right|^{2} \frac{d b d a}{a^{2}} & =\int_{\mathbb{R}_{0}} \int_{\mathbb{R}}\left|\left(D^{-a} \psi * \bar{\psi}\right)(b)\right|^{2} \frac{d b d a}{a^{2}} \\
& =2 \pi \int_{\mathbb{R}_{0}} \int_{\mathbb{R}}\left|\left(D^{-a} \psi\right)^{\wedge}(\varrho) \cdot \hat{\bar{\psi}}(\varrho)\right|^{2} d \varrho \frac{d a}{a^{2}} \\
& =2 \pi \int_{\mathbb{R}_{0}} \int_{\mathbb{R}}|a||\hat{\psi}(-a \varrho)|^{2}|\hat{\bar{\psi}}(\varrho)|^{2} d \varrho \frac{d a}{a^{2}} \\
& =2 \pi\left\|_{\|} \psi\right\|_{0} \cdot \int_{\mathbb{R}} \frac{|\hat{\psi}(\omega)|^{2}}{|\omega|} d \omega
\end{aligned}
$$

In the last step we substituted $\omega=-a \varrho$ an changed the order of integration. As a consequence we can characterize the admissible functions.

Lemma 2.2. $\psi \in L_{2}(\mathbb{R}, d t) \backslash\{0\}$ is admissible if and only if the integral

$$
\int_{\mathbb{R}_{0}} \frac{|\hat{\psi}(\omega)|^{2}}{|\omega|} d \omega
$$

exists.

Remark. As a necessary condition on the admissibility of an element $\psi \in L_{2}(\mathbb{R}, d t)$ we derive

$$
\hat{\psi}(0)=\frac{1}{\sqrt{2 \pi}} \int_{\mathbb{R}} \psi(t) d t=0 ;
$$

i.e. the mean value of $\psi$ has to be zero, if the integral exists (e.g. if $\psi$ is in addition integrable). We call an admissible function also analyzing resp. basic wavelet or wavelet in short. 
Theorem 2.3. Let $\psi$ be admissible and $f \in L_{2}(\mathbb{R}, d t)$, Let $C_{\psi}=2 \pi \int_{\mathbb{R}_{0}} \frac{|\hat{\psi}(\omega)|^{2}}{|\omega|} d \omega$. The integral

$$
\begin{aligned}
L_{\psi} f(b, a) & =\frac{1}{\sqrt{C_{\psi}}}\langle f, U(b, a) \psi\rangle_{0} \\
& =\frac{1}{\sqrt{C_{\psi}}} \frac{1}{\sqrt{|a|}} \int_{\mathbb{R}} \bar{\psi}\left(\frac{t-b}{a}\right) f(t) d t
\end{aligned}
$$

defines an element of $L_{2}\left(\mathbb{R} \times \mathbb{R}_{0}, \frac{d b d a}{a^{2}}\right)$.

Moreover $L_{\psi}: L_{2}(\mathbb{R}, d t) \rightarrow L_{2}\left(\mathbb{R} \times \mathbb{R}_{0}, \frac{d b d a}{a^{2}}\right)$ is an isometry.

Proof. $L_{\psi} f(b, a)$ exists for any $(b, a) \in \mathbb{R} \times \mathbb{R}_{0}$ because $f$ and $T^{b} D^{a} \psi$ are in $L_{2}(\mathbb{R}, d t)$. A similar calculation to (9) results in

$$
\begin{aligned}
\left\|L_{\psi} f\right\|^{2} & =\int_{\mathbb{R}_{0}} \int_{\mathbb{R}}\left|L_{\psi} f(b, a)\right|^{2} \frac{d b d a}{a^{2}} \\
& =\frac{1}{C_{\psi}} \int_{\mathbb{R}_{0}} \int_{\mathbb{R}}|\langle U(b, a) \psi, f\rangle|^{2} \frac{d b d a}{a^{2}} \\
& =\frac{1}{C_{\psi}} \cdot\|f\|_{0}^{2} \cdot 2 \pi \cdot \int_{\mathbb{R}_{0}} \frac{|\hat{\psi}(\omega)|^{2}}{|\omega|} d \omega=\|f\|_{0}^{2} .
\end{aligned}
$$

Definition 2.4. The operator $L_{\psi}: L_{2}(\mathbb{R}, d t) \rightarrow L_{2}\left(\mathbb{R} \times \mathbb{R}_{0}, \frac{d b d a}{a^{2}}\right)(\psi$ admissible $)$ is called wavelet transform with analyzing (basic) wavelet $\psi$.

\section{Extension to Sobolev spaces}

In this section we extend the wavelet transform, which we defined on $L_{2}(\mathbb{R}, d t)$, to Sobolev spaces $H^{\alpha}(\mathbb{R})$ and interpret its images as elements of the fiber space $L_{2}\left(\left(\mathbb{R}_{0}, \frac{d a}{a^{2}}\right), H^{\alpha}(\mathbb{R})\right)$ abbreviated by $\mathscr{F}^{\alpha}$ which is isomorphic to the tensor product $L_{2}\left(\mathbb{R}_{0}, \frac{d a}{a^{2}}\right) \hat{\otimes} H^{\alpha}(\mathbb{R})$ as well as to the Sobolev space with two variables $H^{0, \alpha}\left(\mathbb{R}^{2}, \frac{d a d b}{a^{2}}\right)$, see [1], Chapter 12, pp. 274-279.

If $\mu$ is a measure on $\mathbb{R}_{0}$ and $(B,\|\cdot\|)$ an arbitrary normed space then $L_{2}\left(\left(\mathbb{R}_{0}, d \mu(x)\right),(B,\|\cdot\|)\right)$ consists of those $\phi \in B$ which depend on a real variable and for which holds

$$
\int_{\mathbb{R}_{0}}\|\phi(x)\|^{2} d \mu(x)<\infty .
$$

$H^{\alpha}(\mathbb{R}), \alpha \in \mathbb{R}$, denotes the Sobolev space of those tempered distributions $\gamma$ having a regular and with respect to the weight $\left(1+\omega^{2}\right)^{\alpha}$ square integrable Fourier transform $\hat{\gamma}$. We sometimes call elements of $H^{\alpha}(\mathbb{R})$ signals. 
From now on we assume $\psi$ to be admissible and integrable. If $\psi$ and $f$ are real then $L_{\psi} f$ is real. Without loss of generality we assume $\psi$ and $f$ to be real. Under the assumption above we have

$$
\begin{aligned}
L_{\psi} f(b, a) & =\frac{1}{\sqrt{C_{\psi}}}\left\langle T^{b} D^{a} \psi, f\right\rangle_{0} \\
& =\frac{1}{\sqrt{C_{\psi}}}\left(D^{-a} \psi * f\right)(b) .
\end{aligned}
$$

From (6) we obtain the Fourier transform of $L_{\psi}$ with respect to its shift argument

$$
\left(L_{\psi} f(\cdot, a)\right)^{\wedge}(\omega)=\sqrt{\frac{2 \pi}{C_{\psi}}}|a|^{1 / 2} \hat{\psi}(-a \omega) \hat{f}(\omega) .
$$

Fix $a \in \mathbb{R}_{0}$ and let $f \in \mathscr{P}(\mathbb{R})$, the Schwartz space on $\mathbb{R}$. Let us now determine the $H^{\alpha}(\mathbb{R})$-norm of $L_{\psi} f(\cdot, a)$. For that we need an inequality from Fourier analysis

$$
|\hat{\psi}(\omega)| \leqq \frac{1}{\sqrt{2 \pi}}\|\psi\|_{L_{1}}
$$

leading to

$$
\begin{aligned}
\left\|L_{\psi} f(\cdot, a)\right\|_{\alpha}^{2} & =\int_{\mathbb{R}}\left(1+\omega^{2}\right)^{\alpha}\left|\left(L_{\psi} f(\cdot, a)\right)^{\wedge}(\omega)\right|^{2} d \omega \\
& =\frac{2 \pi}{C_{\psi}} \int_{\mathbb{R}}\left(1+\omega^{2}\right)^{\alpha}\left|\left(D^{-a} \psi\right)^{\wedge}(\omega)\right|^{2}|\hat{f}(\omega)|^{2} d \omega \\
& \leqq \frac{1}{C_{\psi}}\left\|D^{-a} \psi\right\|_{L_{1}}^{2} \int_{\mathbb{R}}\left(1+\omega^{2}\right)^{\alpha}|\hat{f}(\omega)|^{2} d \omega \\
& =K(a, \psi) \cdot\|f\|_{\alpha}^{2},
\end{aligned}
$$

where $K(a, \psi)=\frac{1}{C_{\psi}}|a|\|\psi\|_{L_{1}}^{2}$.

The Schwartz space is dense in $H^{x}(\mathbb{R})$. Therefore we are in a position to extend $L_{\psi} f(\cdot, a)$ uniquely for fixed $a$ to a continuous mapping from $H^{\alpha}(\mathbb{R})$ to itself.

Lemma 3.1. The integral operator $L_{\psi}$ with an integrable and admissible $\psi$ is an isometry from $H^{\alpha}(\mathbb{R}), \alpha \in \mathbb{R}$ to the fiber space $\mathscr{F}^{\alpha}$, i.e.

$$
\left\|L_{\psi} f\right\|_{\mathscr{F}^{\alpha}}=\left(\int_{\mathbb{R}}\left\|L_{\psi} f(\cdot, a)\right\|_{\alpha}^{2} \frac{d a}{a^{2}}\right)^{1 / 2}=\|f\|_{\alpha} .
$$

Proof. It suffices to consider $f \in \mathscr{P}(\mathbb{R})$. The result is shown by a straightforward computation.

$$
\begin{aligned}
\left\|L_{\psi} f\right\|_{\mathbb{y}^{\alpha}}^{2} & =\int_{\mathbb{R}_{0}} \int_{\mathbb{R}}\left(1+\omega^{2}\right)^{\alpha}\left|\left(L_{\psi} f(\cdot, a)\right)^{\wedge}(\omega)\right|^{2} d \omega \frac{d a}{a^{2}} \\
& \stackrel{(13)}{=} \frac{2 \pi}{C_{\psi}} \int_{\mathbb{R}_{0}} \int_{\mathbb{R}}\left(1+\omega^{2}\right)^{\alpha}|a||\hat{\psi}(-a \omega)|^{2}|\hat{f}(\omega)|^{2} d \omega \frac{d a}{a^{2}}
\end{aligned}
$$


Substituting $-a \omega=\xi$ and treating $\omega>0$ and $\omega<0$ separately leads to

$$
\begin{aligned}
\left\|L_{\psi} f\right\|_{\dot{F}^{2}}^{2} & =\frac{2 \pi}{C_{\psi}} \int_{\mathbb{R}_{0}} \frac{|\hat{\psi}(\xi)|^{2}}{|\xi|} d \xi \cdot \int_{\mathbb{R}}\left(1+\omega^{2}\right)^{\alpha}|\hat{f}(\omega)|^{2} d \omega \\
& =\|f\|_{\alpha}^{2} .
\end{aligned}
$$

The signal $f$ and its wavelet transform $L_{\psi} f$ share the same Sobolev order. For a linear isometry $U$ between Hilbert spaces we have that, see [14],

$$
\begin{gathered}
U^{*} U=\text { id and } U U^{*} \text { is the orthogonal projection } \\
\text { onto range }(U) \text { (which is closed), }
\end{gathered}
$$

where $U^{*}$ is the adjoint operator of $U$. From statement (15) it follows immediately that

$$
\text { the transform } L_{\psi} \text { is inverted, on its range, by its adjoint } L_{\psi}^{*}
$$

and that

$$
\text { an element } g \in \mathscr{F}^{\alpha} \text { lies in range }\left(L_{\psi}\right) \text { if and only if } L_{\psi} L_{\psi}^{*} g=g \text {. }
$$

Next we figure out an explicit expression for $L_{\psi}^{*}: \mathscr{F}^{\alpha} \rightarrow H^{\alpha}$. In what follows we use $f \in \mathscr{S}(\mathbb{R}), \quad g(x, a)=g_{1}(x) \cdot g_{2}(a)$ with $g_{1} \in \mathscr{P}(\mathbb{R}), \quad g_{2} \in C_{0}^{\infty}\left(\mathbb{R}_{0}\right)$ and $\Lambda(a, \omega, \alpha)=\left(1+\omega^{2}\right)^{\alpha}\left(L_{\psi} f(\cdot, a)\right)^{\wedge}(\omega) \cdot(g(\cdot, a)){ }^{\wedge}(\omega)$. Setting up a scalar product on $\mathscr{F}^{\alpha}$ in a canonical manner,

$$
(\varrho, \gamma)_{\alpha}=\int_{\mathbb{R}_{0}}\langle\varrho(\cdot, a), \gamma(\cdot, a)\rangle_{\alpha} \frac{d a}{a^{2}}
$$

we get

$$
\left(L_{\psi} f, g\right)_{\alpha}=\int_{\mathbb{R}_{0}} \int_{\mathbb{R}} \Lambda(a, \omega, \alpha) d \omega \frac{d a}{a^{2}} .
$$

Applying two times the Cauchy-Schwarz (C.S.) inequality leads to

$$
\begin{aligned}
\int_{\mathbb{R}_{0}} \int_{\mathbb{R}}|\Lambda(a, \omega, \alpha)| d \omega \frac{d a}{a^{2}} & \leqq \int_{\mathbb{R}_{0}}\left\|L_{\psi} f(\cdot, a)\right\|_{\alpha}\|g(, a)\|_{\alpha} \frac{d a}{a^{2}} \\
& \leqq\left\|L_{\psi} f\right\|_{\mathscr{F}^{\alpha}}\|g\|_{\mathscr{F}^{*}}
\end{aligned}
$$

which allows to change the order of integration in (18).

$$
\left(L_{\psi} f, g\right)_{\alpha}=\int_{\mathbb{R}}\left(1+\omega^{2}\right)^{\alpha} \hat{f}(\omega) \int_{\mathbb{R}_{0}} \sqrt{\frac{2 \pi}{C_{\psi}}}\left(D^{-a} \psi\right)^{\wedge}(\omega)(g(\cdot, a))^{\wedge}(\omega) \frac{d a}{a^{2}} d \omega
$$

We abbreviate the inner integral by $(\tilde{A} g)(\omega)$ and estimate $|\tilde{A} g|$ to conclude that $\tilde{A} g \in L_{2}(\mathbb{R}, d t):$

$$
|\tilde{A} g(\omega)|^{2} \leqq \int_{\mathbb{R}_{0}}\left|(g(\cdot, a))^{\wedge}(\omega)\right|^{2} \frac{d a}{a^{2}}
$$


Again we used the C.S. inequality and get

$$
\begin{aligned}
\int_{\mathbb{R}}|\tilde{A} g(\omega)|^{2} d \omega & \leqq \int_{\mathbb{R}} \int_{\mathbb{R}_{0}}\left|(g(\cdot, a))^{\wedge}(\omega)\right|^{2} \frac{d a}{a^{2}} d \omega \\
& =\|g\|_{\dot{m} 0}^{2} .
\end{aligned}
$$

Consequently there exists a $A g \in L_{2}(\mathbb{R}, d t)$ with

$$
(A g)^{\wedge}(\omega)=\tilde{A} g(\omega)
$$

and now the equation (19) reads as

$$
\begin{aligned}
\left(L_{\psi} f, g\right)_{\alpha} & =\int_{\mathbb{R}}\left(1+\omega^{2}\right)^{\alpha} \hat{f}(\omega)(A g)^{\wedge}(\omega) d \omega \\
& =\langle f, A g\rangle_{\alpha} .
\end{aligned}
$$

In the last step we determine $A g(x)$ using the fact that the integral

$$
\int_{\mathbb{R}_{0}} \int_{\mathbb{R}}\left|\left(D^{-a} \psi\right)^{\wedge}(\omega)(g(\cdot, a))^{\wedge}(\omega)\right| d \omega \frac{d a}{a^{2}}
$$

exists.

$$
\begin{aligned}
A g(x)= & \frac{1}{\sqrt{2 \pi}} \int_{\mathbb{R}}(A g)^{\wedge}(\omega) \mathrm{e}^{\mathrm{i} \omega x} d \omega \\
& =\frac{1}{\sqrt{C_{\psi}}} \int_{\mathbb{R}_{0}} \frac{1}{\sqrt{2 \pi}} \int_{\mathbb{R}}\left(D^{-a} \psi * g(\cdot, a)\right)^{\wedge}(\omega) \mathrm{e}^{\mathrm{i} \omega x} d \omega \frac{d a}{a^{2}} \\
& =\frac{1}{\sqrt{C_{\psi}}} \int_{\mathbb{R}_{0}}\left(D^{-a} \psi * g(\cdot, a)\right)(x) \frac{d a}{a^{2}} \\
& =\frac{1}{\sqrt{C_{\psi}}} \int_{\mathbb{R}_{0}} \int_{\mathbb{R}} \frac{1}{\sqrt{|a|}} \psi\left(\frac{b-x}{a}\right) g(b, a) \frac{d b d a}{a^{2}}
\end{aligned}
$$

We showed that the operators $L_{\psi}$ and $A$ are adjoints of each other on prehilbert spaces of $H^{\alpha}$ resp. $\mathscr{F}^{\alpha}$. This property is inherited by their extensions. Accordingly the extension of $A$ on $\mathscr{F}^{\alpha}$ is identical to $L_{\psi}^{*}$. The abstract characterization (17) of range $\left(L_{\psi}\right)$ results in

Lemma 3.2. Range $\left(L_{\psi}\right) \subset \mathscr{F}^{\alpha}$ is a Hilbert space with reproducing kernel

$$
\begin{gathered}
P(\tilde{b}, \tilde{a}, b, a)=\frac{1}{\sqrt{C_{\psi}}}\left(L_{\psi} \psi\right)\left(\frac{\tilde{b}-b}{a}, \frac{\tilde{a}}{a}\right) . \\
g \in \operatorname{range}\left(L_{\psi}\right) \Leftrightarrow g(\tilde{b}, \tilde{a})=\int_{\mathbb{R}_{0}} \int_{\mathbb{R}} P(\tilde{b}, \tilde{a}, b, a) g(b, a) \frac{d b d a}{a^{2}}
\end{gathered}
$$

Proof. A direct calculation of $L_{\psi} L_{\psi}^{*}$ proves the lemma.

We will now determine the $H^{s}$-distance of two wavelet transforms with different basic wavelets and different argument functions to study the dependence of the transform on its wavelet and its argument. 
Lemma 3.3. For admissible and integrable $\psi, \gamma$ and $f, g \in H^{s}(\mathbb{R}), s \in \mathbb{R}$, holds:

$$
\left\|L_{\psi} f(\cdot, a)-L_{\gamma} g(\cdot, a)\right\|_{s} \leqq \sqrt{|a|}\left(\left\|\frac{\psi}{\sqrt{C_{\psi}}}-\frac{\gamma}{\sqrt{C_{\gamma}}}\right\|_{L_{1}}\|f\|_{s}+\left\|\frac{\gamma}{\sqrt{C_{\gamma}}}\right\|\left\|_{L_{1}}\right\| f-g \|_{s}\right) .
$$

Proof.

$$
\begin{aligned}
\left\|L_{\psi} f(\cdot, a)-L_{\gamma} g(\cdot, a)\right\|_{s} \leqq\left\|L_{\psi} f(\cdot, a)-L_{\gamma} f(\cdot, a)\right\|_{s}+\left\|L_{\gamma} f(\cdot, a)-L_{\gamma} g(\cdot, a)\right\|_{s} \\
=\left(2 \pi \int_{\mathbb{R}}\left(1+\omega^{2}\right)^{s}|\hat{f}(\omega)|^{2}\left|\frac{\left(D^{-a} \psi\right)^{\wedge}(\omega)}{\sqrt{C_{\psi}}}-\frac{\left(D^{-a} \gamma\right)^{\wedge}(\omega)}{\sqrt{C_{\gamma}}}\right|^{2} d \omega\right)^{1 / 2} \\
\quad+\left(2 \pi \int_{\mathbb{R}}\left(1+\omega^{2}\right)^{s}|\hat{f}(\omega)-\hat{g}(\omega)|^{2}\left|\frac{\left(D^{-a} \gamma\right)^{\wedge}(\omega)}{\sqrt{C_{\gamma}}}\right|^{2} d \omega\right)^{1 / 2}
\end{aligned}
$$

Performing the same steps as in (14) to each term of the sum yields the lemma.

A direct application of Lemma 3.3 gives

Corollary 3.4. Let $\psi$ and $f$ be as in the preceding lemma. Then

$$
\left\|L_{\psi} f(\cdot, a)\right\|_{s}=0(\sqrt{|a|}) .
$$

\section{Asymptotic behaviour for small dilation parameters}

We adopt the assumptions on $f$ and $\psi$ from the last paragraph. In addition we assume without loss of generality $\hat{\psi}$ to be real because the admissibility condition is valid not only for the real but also for the imaginary part of $\hat{\psi}[8]$. Then

$$
\begin{aligned}
L_{\psi} f(b, a) & =\frac{1}{\sqrt{C_{\psi}}} \frac{1}{\sqrt{|a|}} \int_{\mathbb{R}} \psi\left(\frac{t-b}{a}\right) f(t) d t \\
& =\frac{1}{\sqrt{C_{\psi}}} \sqrt{|a|} \int_{\mathbb{R}} \hat{\psi}(a \omega) \hat{f}(\omega) \mathrm{e}^{-\mathrm{i} b \omega} d \omega
\end{aligned}
$$

is even in the second variable because $\hat{\psi}$ is. We restrict ourselves to the half-plane $a>0$.

Considering (26) we realize that the integral expression looks like the $\psi$-average $\psi_{a} * f$ of $f$ with $\psi_{a}(x)=a^{-1} \cdot \psi\left(a^{-1} x\right)$.

Indeed we have

$$
\left(\psi_{a} * f\right)(b)=\sqrt{\frac{C_{\psi}}{a}} L_{\psi} f(b,-a)=\sqrt{\frac{C_{\psi}}{a}} L_{\psi} f(b, a) .
$$

For $\psi \in L_{1}(\mathbb{R})$ (i.e. $\psi$ is integrable) with $\int_{\mathbb{R}} \psi(t) d t=1$ the $\psi$-average of $f$ converges to $f$ in the $L_{2}$-norm which means that

$$
\lim _{a \rightarrow 0}\left\|\psi_{a} * f-f\right\|_{0}=0 .
$$

Unfortunately a basic wavelet has zero mean and therefore (28) does not hold for the WT. 
Now we are interested whether an asymptotic behaviour like (28) is possible under certain assumptions on the analyzing wavelet.

For the $\psi$-average of $f$ we write $\Lambda_{\psi} f(\cdot, \cdot)$, i.e.

$$
\Lambda_{\psi} f(b, a)=\left(\psi_{a} * f\right)(b)=\frac{1}{a} \int_{\mathbb{R}} \psi\left(\frac{b-t}{a}\right) f(t) d t .
$$

Lemma 4.1. Let $f \in H^{s}(\mathbb{R}), s \in \mathbb{R}$. Let $\psi \in L_{1}(\mathbb{R})$ with $\int_{\mathbb{R}} \psi(t) d t=1$. Then we have

(i) $\Lambda_{\psi} f(\cdot, a) \rightarrow f(\cdot)$ in $H^{s}(\mathbb{R})$ as $a \rightarrow 0$,

(ii) $d^{k}\left(\Lambda_{\psi} f\right)(\cdot, a)=\Lambda_{\psi}\left(d^{k} f\right)(\cdot, a)=a^{-k}\left(\Lambda_{d^{k} \psi} f\right)(\cdot, a)$, if $d^{k} \psi \in L_{1}(\mathbb{R})$.

( $d^{k}$ denoting the $k$-th generalized derivative).

Proof.

$$
\left\|\psi_{a} * f-f\right\|_{s}^{2}=\int_{\mathbb{R}} I(a, \omega) d \omega
$$

where

$$
I(a, \omega)=\left(1+\omega^{2}\right)^{s}|\hat{f}(\omega)|^{2}|1-\sqrt{2 \pi} \hat{\psi}(a \omega)|^{2} .
$$

With $\quad M=\sup _{\omega \in \mathbb{R}}|1-\sqrt{2 \pi} \hat{\psi}(a \omega)|^{2}$ which exists by the lemma of Riemann-Lebesgue and is independent of $a$ we find

as well as

$$
I(a, \omega) \leqq M \cdot\left(1+\omega^{2}\right)^{s}|\hat{f}(\omega)|^{2}
$$

$$
\lim _{a \rightarrow 0} I(a, \omega)=0 \text { a.e. . }
$$

Applying the dominated convergence theorem yields the assertion.

(ii) Let $\left\{f_{n}\right\}_{n \in \mathbb{N}} \subset \mathscr{S}(\mathbb{R})$ converge to $f$ in $H^{s}(\mathbb{R})$. The equality $d^{k}\left(\Lambda_{\psi} f_{n}\right)=\Lambda_{\psi} d^{k} f_{n}=a^{-k} \Lambda_{d^{k} \psi} f_{n}$ is valid in $H^{s-k}(\mathbb{R})$. Since the operators $A_{\psi}$ and $d^{k}$ are continuous, the limits of the three terms are equal.

Lemma 4.2. Let $0 \not \equiv \varrho \in H^{\beta}(\mathbb{R}), \beta \geqq 1$.

Then $d^{k} \varrho$ is admissible for $1 \leqq k \leqq \beta$.

Proof. First, it is easy to see that $d^{k} \varrho$ is equal to zero if and only if $\varrho \equiv 0$ because zero is the only constant in $H^{s}(\mathbb{R}), s \in \mathbb{R}$. Therefore we have $d^{k} \varrho \neq 0$.

Second, $\beta-k \geqq 0$ implies $d^{k} \varrho \in L_{2}(\mathbb{R})$.

Third, we use the relation

$$
\left(d^{k} \varrho\right)^{\wedge}(\cdot)=\mathrm{i}^{k}(\cdot)^{k} \varrho(\cdot)
$$

to estimate

$$
\begin{aligned}
\int_{\mathbb{R}_{0}} \frac{\left|\left(d^{k} \varrho\right)^{\wedge}(\omega)\right|^{2}}{|\omega|} d \omega & =\int_{\mathbb{R}_{0}}|\omega|^{2 k-1}|\hat{\varrho}(\omega)|^{2} d \omega \\
& \leqq \int_{\mathbb{R}}\left(1+\omega^{2}\right)^{k-1 / 2}|\hat{\varrho}(\omega)|^{2} d \omega \leqq\|\varrho\|_{\beta}^{2} .
\end{aligned}
$$

The result follows from Lemma 2.2 .

Our investigations now focus on the WT with analyzing wavelet $d^{k} \psi \in L_{1}(\mathbb{R})$ with $\psi \in H^{\beta}(\mathbb{R}) \cap L_{1}(\mathbb{R}), \beta \geqq 1$, and $\int_{\mathbb{R}} \psi(t) d t=1$ (thus $\psi$ itself is not admissible). 
Theorem 4.3. Let $f \in H^{s}(\mathbb{R}), s \in \mathbb{R}$, and $\psi \in H^{\beta}(\mathbb{R}) \cap L_{1}(\mathbb{R}), \beta \in \mathbb{N}$, with $\int_{\mathbb{R}} \psi(t) d t=1$ and $d^{k} \psi \in L_{1}(\mathbb{R})$ at least for one $k \in\{1, \ldots, \beta\}$. Then

$$
\lim _{a \rightarrow 0}\left\|\frac{1}{a^{k+1 / 2}} L_{d^{k} \psi} f(\cdot, a)-\frac{1}{\sqrt{C_{k}}} d^{k} f(\cdot)\right\|_{s-k}=0,
$$

where $C_{k}$ abbreviates $C_{d^{k} \psi}$.

Proof. $\psi$ is not identical to zero. According to Lemma $4.2 d^{k} \psi$ is admissible. With an application of Lemma 4.1 (ii) we restate

Now we estimate

$$
\begin{aligned}
L_{d^{k} \psi} f(b, a) & =\sqrt{\frac{a}{C_{k}}} \Lambda_{d^{k} \psi} f(b, a) \\
& =\frac{a^{k+1 / 2}}{\sqrt{C_{k}}} \Lambda_{\psi} d^{k} f(b, a) \\
& =\frac{a^{k+1 / 2}}{\sqrt{C_{k}}} d_{b}^{k}\left(\Lambda_{\psi} f\right)(b, a) .
\end{aligned}
$$

$$
\begin{aligned}
\left\|\frac{1}{a^{k+1 / 2}} L_{d^{k} \psi} f(\cdot, a)-\frac{1}{\sqrt{C_{k}}} d^{k} f(\cdot)\right\|_{s-k} & =\frac{1}{\sqrt{C_{k}}}\left\|d^{k}\left[\Lambda_{\psi} f(\cdot, a)-f(\cdot)\right]\right\|_{s-k} \\
& \leqq \frac{1}{\sqrt{C_{k}}}\left\|\Lambda_{\psi} f(\cdot, a)-f(\cdot)\right\|_{s}
\end{aligned}
$$

using the boundedness of the differential operator from $H^{s}$ to $H^{s-k}$. The term on the right tends to zero by part (i) of Lemma 4.1. This ends the proof.

Remarks.

(i) For $s>\frac{1}{2}+k$ we have uniform convergence. This results immediately by Sobolev's Imbedding Theorem [13].

(ii) For compactly supported $\psi$ we know that $L_{d^{k} \psi} f(\cdot, a) \in H^{s-k+\beta}(\mathbb{R})$ if $f \in H^{s}(\mathbb{R}), \psi \in H^{\beta}(\mathbb{R})$, and $1 \leqq k \leqq \beta$ ( $\beta$ integral). Hence, in accordance with the theorem above, $\frac{1}{a^{k+1 / 2}} L_{d^{k} \psi} f(\cdot, a)$ is an approximation of $\frac{1}{\sqrt{C_{k}}} d^{k} f$ which is at least
$\beta$ levels smoother than its limit.

\subsection{Local convergence}

In practical applications of the WT, i.e. the analysis and synthesis of timedependent signals, the signal $f$ is compactly supported. Even if this signal possesses a high order of smoothness within its support under a global viewpoint we can only deduce that $f$ is square integrable over the real line, which means $f \in H^{\circ}(\mathbb{R})$.

By Theorem $4.3 \frac{\sqrt{C_{k}}}{a^{k+1 / 2}} L_{\mathbf{d}^{k} \psi} f$ approximates the $k$-th derivative of $f$ only in $H^{-k}(\mathbb{R})$ although $f$ is local an element of the Sobolev space $H^{s}(\mathbb{R})$ with $s>0$ and therefore we would expect a kind of local convergence in the stronger norm of $H^{s-k}(\mathbb{R})$.

We specify the concept of local convergence. Therefore we define the local Sobolev spaces [13]. 
Definition 4.4. Let $\Omega \subset \mathbb{R}$ be open.

$$
\begin{aligned}
H_{\text {loc }}^{s}(\Omega):=\{f \text { is a distribution } \mid & \forall \Omega^{\prime} \subset \Omega, \Omega^{\prime} \text { compact, } \\
& \left.\exists g_{\Omega^{\prime}} \in H^{s}(\mathbb{R}): f \equiv g_{\Omega^{\prime}} \text { on } \Omega^{\prime}\right\}
\end{aligned}
$$

is called local Sobolev space of order s.

The

Lemma 4.5. $f \in H_{\mathrm{loc}}^{s}(\Omega) \Leftrightarrow f \cdot \Phi \in H^{s}(\mathbb{R}) \forall \Phi \in C_{0}^{\infty}(\Omega)$

$\left(C_{0}^{\infty}(\Omega)\right.$ denotes the space of the test functions with compact support in $\Omega$ )

suggests a concept of convergence in $H_{\mathrm{loc}}^{s}(\Omega)$.

Definition 4.6. Let $\left\{f_{n}\right\}_{n \in \mathbb{N}}$ be a sequence in $H_{\mathrm{loc}}^{s}(\Omega)$ and $f \in H_{\mathrm{loc}}^{s}(\Omega)$. $\left\{f_{n}\right\}_{n \in \mathbb{N}}$ converges to $f$ in $H_{\text {loc }}^{s}(\Omega)$ (local convergence) if and only if $\left\|\Phi f_{n}-\Phi f\right\|_{s}$ converges to zero for any $\Phi \in C_{0}^{x}(\Omega)$.

Remark. This concept of local convergence is well defined because the limit is uniquely determined.

Without loss of generality we assume that

$$
\operatorname{supp}(f)=[-T, T]=I .
$$

Further we consider

$$
\left.f \in H_{\mathrm{loc}}^{s}\left(I^{0}\right) \quad \text { with } I^{0}=\right]-T, T[\text { and } s \in \mathbb{R} .
$$

For $0<\varepsilon<T$ let $J_{\varepsilon}$ be the compact interval $[-\varepsilon, \varepsilon]$. We know from real analysis that there is a $\Gamma_{\varepsilon} \in C_{0}^{\infty}\left(I^{0}\right)$ which is identical to 1 on $J_{\varepsilon}$.

\section{Lemma 4.7}

(i) $\Gamma_{\varepsilon}(\cdot) f(\cdot) \in H^{s}(\mathbb{R})$

(ii) $\Gamma_{\varepsilon}(\cdot) f(\cdot)$ converges to $f$ in $H_{\mathrm{loc}}^{s}\left(I^{0}\right)$ as $\varepsilon$ tends to $T$.

(iii) $\Gamma_{\varepsilon}(\cdot) f(\cdot) \equiv f(\cdot)$ on $H_{\mathrm{loc}}^{s}\left(J_{\varepsilon}^{0}\right)$.

Proof. (i) is the statement of Lemma 4.5.

(ii) Let $\Phi \in C_{0}^{\infty}\left(I^{0}\right)$. For sufficiently large $\varepsilon_{\Phi}$ with $0<\varepsilon_{\Phi}<T$ we have $\operatorname{supp}(\Phi) \subseteq J_{\varepsilon}$ for all $\varepsilon$ with $\varepsilon_{\Phi} \leqq \varepsilon<T$.

This implies $\phi \Gamma_{\varepsilon} f=\Phi f$ in $H^{s}(\mathbb{R})$ for $\varepsilon_{\Phi} \leqq \varepsilon<T$ and thus the assertion.

(iii) It is clear that both $\Gamma_{\varepsilon} f$ and $f$ are in $H_{\mathrm{loc}}^{s}\left(J_{\varepsilon}^{0}\right)$. We still have to show the equality. Let a test function $\Phi \in C_{0}^{\infty}\left(J_{\varepsilon}^{0}\right)$ act on the distribution $\Gamma_{\varepsilon} f$ :

$$
\left(\Gamma_{\varepsilon}(t) f(t), \Phi(t)\right)=\left(f(t), \Gamma_{\varepsilon}(t) \Phi(t)\right)=(f(t), \Phi(t)) .
$$

A local version of Theorem 4.3 reads as

Theorem 4.8. Let $f$ fulfill (30) and (31). Let $\psi$ be defined as in Theorem 4.3 and $\Gamma_{\varepsilon}$ as above.

Then $\frac{1}{a^{k+1 / 2}} L_{d^{k} \psi}\left(\Gamma_{\varepsilon} f\right)(\cdot, a)$ converges to $\frac{1}{\sqrt{C_{k}}} d^{k} f$ in $H_{\text {loc }}^{s-k}\left(J_{\varepsilon}^{0}\right)$ for any $\varepsilon \in] 0, T[$ as a tends to zero.

Remark. Even locally we can reach the convergence in the strongest norm. 
Proof of the theorem. First we conclude $d^{k} f=d^{k}\left(\Gamma_{\varepsilon} f\right)$ in $H_{\mathrm{loc}}^{s-k}\left(J_{\varepsilon}^{0}\right)$ with the Leibniz rule and the action of $\Phi \in C_{0}^{\infty}\left(J_{\varepsilon}^{0}\right)$ on $d^{k}\left(\Gamma_{\varepsilon} f\right)$ :

$$
\left(d^{k}\left(\Gamma_{\varepsilon} f\right), \Phi\right)=\sum_{i=0}^{k}\left(\begin{array}{l}
k \\
i
\end{array}\right)\left(d^{k-i} f,\left(d^{i} \Gamma_{\varepsilon}\right) \Phi\right)=\left(d^{k} f, \Phi\right) .
$$

The last equality holds true because $\left.\Gamma_{\varepsilon}\right|_{\operatorname{supp}(\Phi)} \equiv 1$.

Theorem 4.3 yields

$$
\frac{1}{a^{k+1 / 2}} L_{d^{k} \psi}\left(\Gamma_{\varepsilon} f\right)(\cdot, a) \stackrel{a \rightarrow 0}{\longrightarrow} \frac{1}{\sqrt{C_{k}}} d^{k}\left(\Gamma_{\varepsilon} f\right)(\cdot) \in H^{s-k}(\mathbb{R}) .
$$

To continue the proof we need the boundedness of the multiplication operator on $H^{\alpha}$. Let $\Pi \in \mathscr{S}(\mathbb{R})$ and let $T_{\Pi}: H^{\alpha} \rightarrow H^{\alpha}$ be defined by $T_{\Pi} f=\Pi \cdot f$. Then $T_{I I}$ is continuous for all $\alpha \in \mathbb{R}[13]$. We are now able to prove the desired convergence in $H_{\mathrm{doc}}^{s-k}\left(J_{\varepsilon}^{0}\right)$.

Let $\Phi \in C_{0}^{\infty}\left(J_{\varepsilon}^{0}\right) \subset \mathscr{S}(\mathbb{R})$ :

$$
\begin{aligned}
& \left\|\Phi(\cdot) \frac{1}{a^{k+1 / 2}} L_{d^{k} \psi}\left(\Gamma_{\varepsilon} f\right)(\cdot, a)-\Phi(\cdot) \frac{1}{\sqrt{C_{k}}} d^{k} f(\cdot)\right\|_{s-k} \\
& \leqq\left\|T_{\Phi}\right\| \cdot\left\|\frac{1}{a^{k+1 / 2}} L_{d^{k} \psi}\left(\Gamma_{\varepsilon} f\right)(\cdot, a)-\frac{1}{\sqrt{C_{k}}} d^{k}\left(\Gamma_{\varepsilon} f\right)(\cdot)\right\|_{s-k} .
\end{aligned}
$$

Under local conditions of smoothness on the signal, statements can be made about the order of convergence.

Lemma 4.9. Let $f$ be two times continuously differentiable in a neighbourhood of $b \in \mathbb{R}$ (e.g. $f \in H_{\mathrm{loc}}^{s}(] b-\varepsilon, b+\varepsilon[)$ for some $\varepsilon>0$ and $\left.s>2+\frac{1}{2}\right)$. Let $\psi \in H^{\beta}(\mathbb{R}) \cap L_{1}(\mathbb{R}), \beta \geqq 1$, with $\int_{\mathbb{R}} \psi(t) d t=1$ and $\operatorname{supp} \psi=\left[T_{1}, T_{2}\right]$. For $a>0$ sufficiently small holds

$$
a^{-3 / 2} L_{d \psi} f(b, a)=\frac{1}{\sqrt{C_{1}}} f^{\prime}(b)+O(a)
$$

(prime indicates the first classical derivative).

Proof. Using the facts that for sufficiently small $a d f$ is equal to $f^{\prime}$ in $\left[b+a T_{1}, b+a T_{2}\right]=: I(b, a)$ and that $M(a)=\sup _{\xi \in I(b, a)}\left|f^{\prime \prime}(\xi)\right|$ exists, we obtain by the mean value theorem

$$
\begin{aligned}
\left|a^{-3 / 2} L_{d \psi} f(b, a)-\frac{1}{\sqrt{C_{1}}} f^{\prime}(b)\right| & =\frac{1}{\sqrt{C_{1}}}\left|\Lambda_{\psi} d f(b, a)-f^{\prime}(b)\right| \\
& \leqq \frac{1}{\sqrt{C_{1}}} \cdot \frac{1}{a} \int_{I(b, a)}\left|\psi\left(\frac{t-b}{a}\right)\right|\left|f^{\prime}(t)-f^{\prime}(b)\right| d t \\
& \leqq \frac{1}{\sqrt{C_{1}}} \cdot \frac{1}{a} \cdot M(a) \int_{I(b, a)}\left|\psi\left(\frac{t-b}{a}\right)\right||t-b| d t \\
& =K(a, b, f) \cdot a
\end{aligned}
$$

with $K(a, b, f)=\frac{1}{\sqrt{C_{1}}} \cdot M(a) \int_{T_{1}}^{T_{2}}|\psi(y)||y| d y$. 


\subsection{Wavelet transform with compactly supported wavelet}

The practical importance of the former statements of this section would be increased if we could find a criterion to decide whether an integrable wavelet $\varrho$ can be represented by $\varrho=d^{k} \psi$ with $\psi \in H^{k}(\mathbb{R}) \cap L_{1}(\mathbb{R})$ and $\int_{\mathbb{R}} \psi(t) d t \neq 0$.

We will see that the compactness of the support of the basic wavelet suffices to guarantee the existence of such a $\psi$.

We need a preparatory lemma which can be verified by induction.

Lemma 4.10. Let $\phi_{l}(x)=\int_{\alpha}^{x} \phi_{l-1}(t) d t, l \geqq 1, x \in[\alpha, \beta]$ a sequence of functions with $\phi_{0} \in L_{1}([\alpha, \beta])$. Then

$$
\phi_{l+1}(x)=\frac{1}{l !} \int_{x}^{x} \phi_{0}(z) \cdot(x-z)^{l} d z
$$

The following theorem and its corollary formulate the above mentioned criterion.

Theorem 4.11. Let $\varrho$ be square integrable and not identical to zero. Let $[\alpha, \beta]$ be the compact support of $\varrho$ and let its mean value be equal to zero.

Then exists a $k \in \mathbb{N}$ and a uniquely determined $\psi \in H^{k}(\mathbb{R}) \cap L_{1}(\mathbb{R})$ with $\int_{\mathbb{R}} \psi(t) d t \neq 0$ and $d^{k} \psi=\varrho$.

Proof. We define a sequence of functions whose $k$-th member is our searched $\psi$ :

$$
\begin{aligned}
\phi_{0}(x) & :=\varrho(x) \\
\phi_{l}(x): & =\int_{\alpha}^{x} \phi_{l-1}(t) d t \quad l=1,2, \ldots
\end{aligned}
$$

$\left\{\phi_{l}\right\}_{l \in \mathbb{N}}$ has the following properties:

(a) $\phi_{l} \in C^{l-1}([\alpha, \beta]), l \geqq 1$,

(b) $\phi_{1}^{\prime}=\varrho$ almost everywhere

( $\phi_{1}$ is absolute continuous),

$\phi_{l}^{(j)}(x)=\phi_{l-j}(x) \forall x \in[\alpha, \beta]$ and $0 \leqq j \leqq l-1$

$\left(\phi^{(j)}\right.$ indicates the $j$-th classical derivative of $\left.\phi\right)$,

(c) $\phi_{l+1}(x)=\frac{1}{l !} \int_{a}^{x} \varrho(z)(x-z)^{l} d z$ (Lemma 4.10).

Assertion. There is a $k \in \mathbb{N}$ with $\phi_{k+1}(\beta)=\int_{\alpha}^{\beta} \phi_{k}(t) d t \neq 0$.

We proof the assertion indirectly. Let us therefore assume $\phi_{I+1}(\beta)=0 \forall l \geqq 0$. Property (c) leads to $0=(1 / l !) \int_{\alpha}^{\beta} \varrho(z)(\beta-z)^{l} d z$ for $l \geqq 0$. This implies $\int_{\alpha}^{\beta} \varrho(z)$ $z^{l} d z=0 \forall l \geqq 0$ which contradicts $\varrho \neq 0$.

The assertion is true and we set

$$
\begin{aligned}
& k:=\min \left\{l \in \mathbb{N} \mid \phi_{l+1}(\beta) \neq 0\right\} \\
& \psi:=\phi_{k}
\end{aligned}
$$

and show that $\psi$ has the desired properties:

(i) $d^{j} \psi=\phi_{k-j}, 0 \leqq j \leqq k$

(ii) $d^{j} \psi(a)=d^{j} \psi(b)=\phi_{k-j}(b)=0,0 \leqq j \leqq k-1$

This means $d^{j} \psi \in C^{k-j-1}(\mathbb{R}), 0 \leqq j \leqq k-1$ with compact support $[\alpha, \beta]$ and $d^{j} \psi \in L_{2}(\mathbb{R})$ for $0 \leqq j \leqq k$, i.e. $\psi \in H^{\bar{k}}(\mathbb{R})$.

(iii) $\int_{\mathbb{R}} \psi(t) d t=\int_{\alpha}^{\beta} \phi_{k}(t) d t=\phi_{k+1}(\beta) \neq 0$. 
Corollary 4.12. Let $0 \not \equiv \varrho \in L_{2}(\mathbb{R})$ be compactly supported and $\int_{\mathbb{R}} \varrho(t) d t=0$. Then $\varrho$ is admissible.

Proof. The preceding theorem supplies a $k \in \mathbb{N}$ and a $\psi \in H^{k}(\mathbb{R})$ with $\psi \neq \equiv 0$, $d^{k} \psi=\varrho$. Lemma 4.2 completes the proof.

Remarks. (a) Within the remark on Lemma 2.2 the mean value condition $\int_{\mathbb{R}} \varrho(t) d t=0$ was mentioned as a necessary condition for the admissibility of a $L_{1}$-function $\varrho$. The corollary states that for $\varrho \neq \equiv 0$ with compact support the mean value condition is also sufficient.

(b) For all WT with compactly supported basic wavelets the statements of convergence described in the Theorems 4.3 resp. 4.8 hold.

\section{Consequences for the discrete wavelet transform}

In this paragraph we want to discuss the conclusions and consequences for the discrete wavelet transform (DWT) arising from Theorems 4.3 and 4.11 .

The DWT is a modification of the continuous one which is relevant and adequate for practical applications like signal theory [6], edge detection, and pattern recognition $[9,10]$.

In the sequel we give a short description of its essential properties:

For suitable grids $G=\left\{\left(b_{m}, a_{n}\right) \mid m, n \in \mathbb{Z}\right\} \subset \mathbb{R} \times \mathbb{R}_{0}$ and suitable basic wavelets $\psi$ the mapping

$$
\begin{aligned}
L_{\psi}^{D}: L_{2}(\mathbb{R}) & \rightarrow l_{2}\left(\mathbb{Z}^{2}\right) \text { defined by } \\
\left(L_{\psi}^{D} f\right)_{m, n} & =\left\langle T^{b_{m}} D^{a_{n}} \psi, f\right\rangle_{0} \\
& =\sqrt{C_{\psi}} L_{\psi} f\left(b_{m}, a_{n}\right)
\end{aligned}
$$

is continuous and continuously invertible.

Thus there exist positive numbers $A$ and $B$ such that

$$
A\|f\|_{0} \leqq\left\|L_{\psi}^{D} f\right\|_{l_{2}} \leqq B\|f\|_{0}
$$

Then the set

$$
R(G, \psi)=\left\{T^{b} D^{a} \psi \mid(b, a) \in G\right\}
$$

is called a frame of $L_{2}(\mathbb{R})$.

We refer the reader to [3] and [4] for sufficient conditions on the grid $G$ and the analyzing wavelet $\psi$ so that $R(G, \psi)$ constitutes a frame.

For sake of simplicity we restrict ourselves to the dyadic grid, see $[2,11]$, $G_{\mathrm{d}}=\left\{\left(2^{m} n, 2^{m}\right) \mid m, n \in \mathbb{Z}\right\}$ and basic wavelets with the following properties:

(P1) $R\left(G_{\mathrm{d}}, \psi\right)=\left\{\psi_{m, n}(x)=2^{-m / 2} \psi\left(2^{-m} x-n\right) \mid m, n \in \mathbb{Z}\right\}$ forms an orthonormal basis (ONB) in $L_{2}(\mathbb{R})$ (called wavelet basis).

(P2) There exists a function $\phi \in L_{2}(\mathbb{R})$ such that $\left\{\phi_{j, k}(x)=2^{-j / 2} \phi\left(2^{-j} x-k\right) \mid k \in \mathbb{Z}\right\}$ forms an ONB for

$$
V_{j}=\underset{j<m}{\oplus} W_{m} \quad \text { with } W_{m}=\overline{\operatorname{span}\left\{\psi_{m, n} \mid n \in \mathbb{Z}\right\}}
$$


The simplest example of a wavelet satisfying (P1) and (P2) is the Haar-function

$$
\psi(x)=\left\{\begin{aligned}
1 & \text { if } 0 \leqq x \leqq \frac{1}{2} \\
-1 & \text { if } \frac{1}{2}<x \leqq 1 \\
1 & \text { else }
\end{aligned}\right.
$$

$R\left(G_{\mathrm{d}}, \psi\right)$ is the Haar-system well known from functional analysis.

The function $\phi(x)=\left\{\begin{array}{ll}1 & \text { if } 0 \leqq x<1 \\ 0 & \text { else }\end{array}\right.$ satisfies (P2) with respect to the Haarwavelet.

Further examples of arbitrarily smooth $\psi$ and $\phi$ can be found in [3].

We mention some properties of the spaces $V_{j}$. Let $P_{j}$ resp. $Q_{j}$ denote the orthogonal projections onto $V_{j}$ resp. $W_{j}$.

$$
\begin{gathered}
\ldots \subset V_{2} \subset V_{1} \subset V_{0} \subset V_{-1} \subset V_{-2} \subset \ldots \\
\cap V_{j}=\{0\}, \quad \overline{U V_{j}}=L_{2}(\mathbb{R}) \\
P_{j} f \rightarrow f \quad \text { as } j \rightarrow-\infty \\
P_{j} f \rightarrow 0 \quad \text { as } j \rightarrow+\infty \\
V_{j-1}=V_{j} \oplus W_{j}, \quad P_{j-1}=P_{j}+Q_{j}
\end{gathered}
$$

In [11] the above introduced construction of the spaces $V_{j}, W_{j}$, and the projections $P_{j}, Q_{j}$ is called multiscale analysis. Because of (36) and (37) $P_{j} f$ is heuristically interpreted as a representation of $f$ on the scale $2^{j} . P_{j} f$ contains only details of $f$ with minimal size $2^{j}$.

So we are able to interpret $Q_{j} f=\sum_{n \in \mathbb{Z}}\left\langle f, \psi_{j, n}\right\rangle_{0} \psi_{j, n}=\left(P_{j-1}-P_{j}\right) f$ as the change of $f$ at the transition from the scale $2^{j-1}$ to the coarser scale $2^{j}$.

The additional requirements ( $\mathrm{P} 1)$ and $(\mathrm{P} 2)$ yield an iterative algorithm (by S. Mallat [11]) for analyzing and synthezing signals. The analysis part is formulated as follows:

With the notations $\left(s \in L_{2}(\mathbb{R})\right)$

$$
c_{k}^{j}(s)=\left\langle s, \phi_{j, k}\right\rangle_{0}
$$

for the moments of $P_{j}$ s relative to $\left\{\phi_{j, k} \mid k \in \mathbb{Z}\right\}$ and

$$
q_{k}^{j}(s)=\left\langle s, \psi_{j, k}\right\rangle_{0}
$$

for the moments (wavelet coefficients) of $Q_{j} f$ relative to $\left\{\psi_{j . k} \mid k \in \mathbb{Z}\right\}$ the Mallatiteration reads

$$
c_{k}^{j}=\sum_{n \in \mathbb{Z}} h(n-2 k) c_{n}^{j-1}
$$

with the filter $h(n)=2^{-1 / 2} \int \phi(x / 2) \phi(x-n) d x$ and

$$
q_{k}^{j}=\sum_{n \in \mathbb{Z}} g(n-2 k) c_{n}^{j-1}
$$

with the filter $g(n)=2^{-1 / 2} \int \psi(x / 2) \phi(x-n) d x$. (41) and (42) are consequently discrete convolutions which can be efficiently calculated by FFT. 
If the signal to analyze is only sampled at discrete times we have to perform some sort of interpolation together with a projection before we can iterate. The smallest detail of the discrete signal, given by the sampling distance, is set equal to one without loss of generality. For obvious reasons we construct the members of the signal sequence $\left\{s_{k}\right\}_{k \in \mathbb{Z}}$ as the moments of a $L_{2}$-function projected onto $V_{0}$. The continuous signal is now $s(x)=\sum_{k} s_{k} \phi(x-k) \in V_{0}$. For $j \geqq 1$ the iteration can be started with $c_{k}^{0}(s)=s_{k}$.

These facts about the DWT should suffice to interpret the orthogonal projection $Q_{j}: L_{2}(\mathbb{R}) \rightarrow W_{j}$ within the framework of Sect. 4.

As mentioned above we obtain from $\left\{f_{k}^{0}\right\}_{k \in \mathbb{Z}}$ the continuous signal $s(x)=\sum_{k} f_{k}^{0} \phi_{0, k}(x)$ in $V_{0}$.

We set $f_{k}^{j}=c_{k}^{j}(s)$ and $q_{k}^{j}=q_{k}^{j}(s)$ and calculate

$$
\begin{aligned}
\sqrt{C_{\psi}}\left(L_{\psi} P_{j} s\right)\left(2^{j+1} k, 2^{j+1}\right) & =2^{-(j+1) / 2} \int_{\mathbb{R}} \psi\left(\frac{x-2^{j+1} k}{2^{j+1}}\right) P_{j} s(x) d x \\
& =\sum_{n \in \mathbb{Z}} f_{n}^{j} \cdot 2^{-1 / 2} \int_{\mathbb{R}} \psi\left(\frac{x}{2}\right) \phi(x-n+2 k) d x \\
& =\sum_{n \in \mathbb{Z}} f_{n}^{j} g(n-2 k) .
\end{aligned}
$$

By (42) we have the equality

$$
q_{k}^{j+1}=\sqrt{C_{k}}\left(L_{\psi} P_{j} s\right)\left(2^{j+1} k, 2^{j+1}\right)
$$

exploiting the wavelet coefficients to be an approximation of

$$
2^{(j+1)(l+1 / 2)} d^{l}\left(P_{j} s\right)\left(2^{j+1} k\right)
$$

if $\psi$ is the $l$-th derivative of a function $\varrho$ fulfilling the hypotheses of Theorem 4.3.

$q_{k}^{j+1}$ is an average of $D 2^{j} \psi$ and $P_{j} s$ at instant $2^{j+1} k$. For decreasing $j$ the average is computed over smaller domains (within Mallet's algorithm for discrete signals the smallest value of $j$ is zero).

For the projections $Q_{j+1}$ this means

$$
\begin{aligned}
Q_{j+1} s(x) & =\sqrt{C_{\psi}} \sum_{k \in \mathbb{Z}}\left(L_{\psi} P_{j} s\right)\left(2^{j+1} k, 2^{j+1}\right) \psi_{j+1, k}(x) \\
& \approx 2^{(j+1)(l+1 / 2)} \sum_{k \in \mathbb{Z}} d^{l}\left(P_{j} s\right)\left(2^{j+1} k\right) \psi_{j+1, k}(x) .
\end{aligned}
$$

Thus $Q_{j+1}$ approximates an interpolation in the sense mentioned above of the sequence $\mathscr{S}_{j}=\left\{2^{(j+1)(l+1 / 2)} d^{l}\left(P_{j} s\right)\left(2^{j+1} k\right) \mid k \in \mathbb{Z}\right\}$ in the space $W_{j+1}$. The $k$-th member of $\mathscr{S}_{j}$ is the $l$-th derivative of the projection of $s \in V_{0}$ onto $V_{j}$ at instant $2^{j+1} k$.

$$
\text { For } j=0 \text { (44) reduces to }
$$

$$
Q_{1} s(x) \approx 2^{(l+1 / 2)} \cdot \sum_{k} s^{(l)}(2 k) \psi(2 x-k) .
$$

Hence $Q_{1} s$ interpolates the $l$-th derivative of $s(x)=\sum_{k} f_{k}^{0} \phi(x-k)$ in $W_{1}$ founded upon the double stepsize of the starting sequence $\left\{f_{k}^{o}\right\}_{k \in \mathbb{Z}}$.

The example of the Haar-wavelet (35) explains in a clear way the meaning of (44) and (45). 
The filters $h$ and $g$ of (41) and (42) are easily computed:

$$
h(n)=\left\{\begin{array}{ll}
2^{-1 / 2} & \text { if } n \in\{0,1\} \\
0 & \text { else }
\end{array}, \quad g(n)=\left\{\begin{array}{cl}
2^{-1 / 2} & \text { if } n=0 \\
-2^{-1 / 2} & \text { if } n=1 . \\
0 & \text { else }
\end{array}\right.\right.
$$

Mallat's iteration reads

$$
\begin{aligned}
f_{k}^{j} & =2^{-1 / 2}\left(f_{2 k}^{j-1}+f_{2 k+1}^{j-1}\right) \\
q_{k}^{j} & =2^{-1 / 2}\left(f_{2 k}^{j-1}-f_{2 k+1}^{j-1}\right)
\end{aligned} \quad j=1,2, \ldots
$$

We write out the first two iterations for the wavelet coefficients

$$
\begin{aligned}
& q_{k}^{1}=2^{-1 / 2}\left(f_{2 k}^{0}-f_{2 k+1}^{0}\right) \\
& q_{k}^{2}=2^{-1}\left(\left(f_{4 k}^{0}+f_{4 k+1}^{0}\right)-\left(f_{4 k+2}^{0}+f_{4 k+3}^{0}\right)\right)
\end{aligned}
$$

and recognize at the first step a difference quotient of the original signal. At the second and all other steps the wavelet coefficients are formed by differences of smoothed versions where averages of neighbouring elements are taken.

The practical importance of the Haar-function to serve as a basic wavelet is therefore very limited particularly in connection with noisy signals since the evaluation of (48) is very instable.

Starting with the filter $h$ of (41) Daubechies [3] gives a method for constructing basic wavelets satisfying the conditions (P1) and (P2). She shows that filters with finite length belong to compactly supported wavelets. In accordance with Theorem 4.11 and the statements of this section the approximations (44) and (45) hold for the corresponding DWT.

General result. The signal changes represented by the DWT at the transition from a finer to a coarser scale are nothing but the jumps in a derivative of a smoothed version resulted by 'projecting the signal onto the coarser scale'.

\section{Numerical examples}

We illustrate the statement of Theorem 4.3 by two examples. The signal under consideration is

$$
f(x)=\left\{\begin{array}{ll}
1 & \text { if } 1<|x| \leqq 1.5 \\
2+x & \text { if }-1 \leqq x<0 \\
2-x & \text { if } 0 \leqq x \leqq 1 \\
0 & \text { else }
\end{array} \quad \in H^{s}(\mathbb{R}), s<\frac{1}{2}\right.
$$

with the derivatives ( $\delta$ is Dirac's distribution)

$$
\begin{gathered}
d f(x)=\delta(x+1.5)-\delta(x-1.5)+\left\{\begin{aligned}
1 & \text { if }-1 \leqq x<0 \\
-1 & \text { if } 0<x \leqq 1 \\
0 & \text { else }
\end{aligned}\right. \\
d^{2} f(x)=\delta^{\prime}(x+1.5)-\delta^{\prime}(x-1.5)+\delta(x+1) \\
-2 \delta(x)+\delta(x-1) .
\end{gathered}
$$


Our first example uses the basic wavelet

$$
\varrho_{1}(x)=\left\{\begin{aligned}
1 & \text { if }-1 \leqq x<0 \\
-1 & \text { if } 0<x \leqq 1 \\
0 & \text { else }
\end{aligned}\right.
$$

which is just a translated and dilated version of the Haar-wavelet (35).

According to Theorems 4.3 and $4.11 \sqrt{C_{\varrho_{1}}} a^{-3 / 2} L_{\varrho_{1}} f(b, a)$ approximates (50) for small $a$ as is shown in Fig. 6.1. The ranges of the shift $b$ and the dilation $a$ are marked. To see more details in the diagram we cut off the values of the transform with modulus greater than 5 .

Figure 6.2 shows $\sqrt{C_{\varrho_{2}}} a^{-5 / 2} L_{\varrho_{2}} f(b, a)$ with analyzing wavelet

$$
\varrho_{2}(x)=\left\{\begin{aligned}
1 & \text { if } 0.5 \leqq|x| \leqq 1 \\
-1 & \text { if }|x|<0.5 \\
0 & \text { else }
\end{aligned}\right.
$$

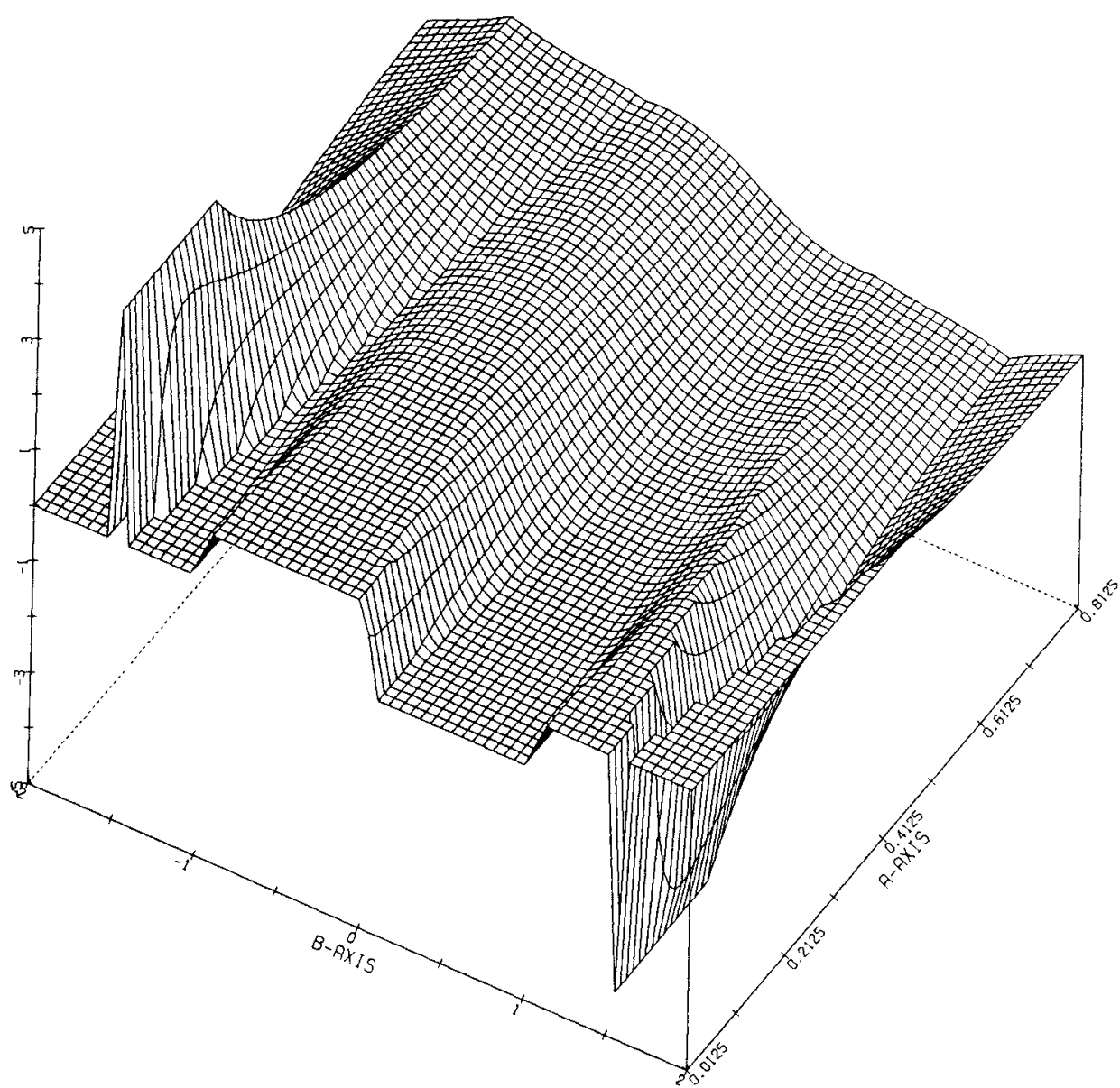

Fig. 6.1. Approximation of (50) by WT 


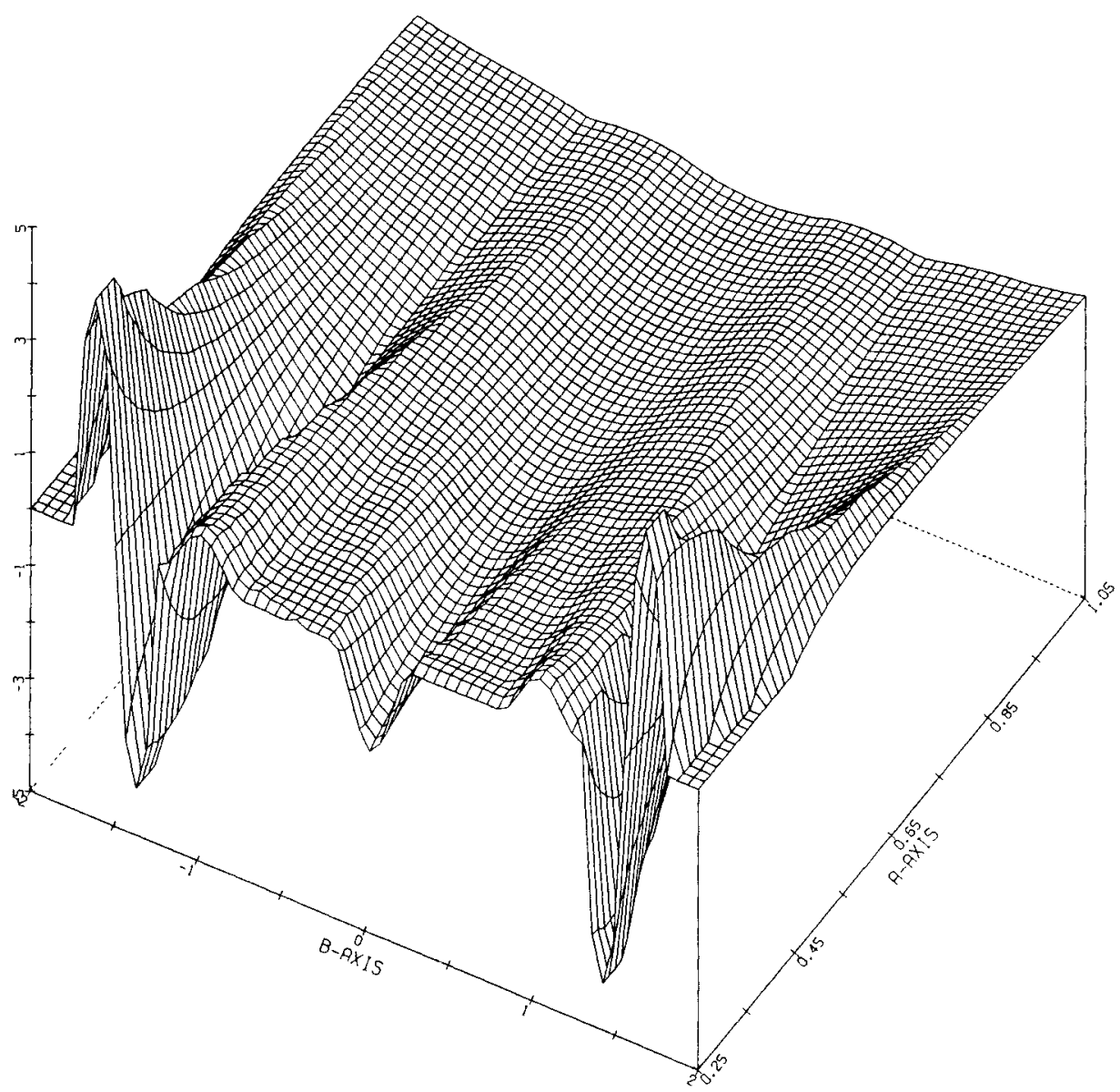

Fig. 6.2. Approximation of (51) by WT

Again the results of the former sections predict convergence to (51). We note a special feature of this example. Due to the ill-posedness of numerical differentiation the errors made by discretization blow up if $a$ approaches zero. To avoid noise amplification we have regularized by limiting $a$ to the interval $[0.25,1.05]$. Therefore the quality of the approximation is worse than the one of the first example.

Acknowledgement. I would like to thank Prof. Dr. A.K. Louis for the fruitful discussions and the helpful suggestions on this paper.

\section{References}

1. Aubin, J.P.: Applied functional analysis, New York: J. Wiley 1979

2. Daubechies, I.: The wavelet transform, time-frequency localisation and signal analysis. IEEE Trans. Autom. Control (to appear) 
3. Daubechies, 1.: Orthonormal bases of compactly supported wavelets. Com. Pure Appl. Math. XL1, 909-996 (1988)

4. Daubechies, I., Grossmann, A., Meyer, M.: Painless nonorthogonal expansions. J. Math. Phys. 27, 1271-1283 (1986)

5. Goupillaud, P., Grossmann, A., Morlet, J.: Cycle-octave and related transforms in seismic signal analysis. Geoexploration 23, 85-102 (1984/85)

6. Grossmann, A., Holschneider, M., Kronland-Martinet, R., Morlet, J:: Detection of abrupt changes in sound signals with the help of wavelet transform. Preprint, Centre de Physique Théorique, CNRS, Marseille (France)

7. Grossmann, A., Morlet, J., Paul, T.: Transforms associated to square integrable group representations I: General results. J. Math. Phys. 26, 2473-2479 (1985)

8. Grossmann, A., Morlet, J., Paul, T.: Transforms associated to square integrable group representations II: Examples. Ann. Inst. Henri Poincaré, 45, 293309 (1986)

9. Kronland-Martinet, R., Morlet, J., Grossmann, A.: Analysis of sound patterns through wavelet transforms. J. Pat. Recog. Art. Intell. 1, $273-302$ (1987)

10. Mallat S.: A theory for multiresolution signal decomposition: The wavelet representation. Preprint GRASP Lab., Dept. of Computer and Information Science, Univ. of Pennsylvania

11. Mallat S.: A theory for multiresolution signal decomposition: The scale change representation. Preprint GRASP Lab., Dept. of Computer and Information Science, Univ. of Pennsylvania

12. Paul T.: Functions analytic on the half-plane as quantum mechanical states. J. Math. Phys. $25,3252-3263$ (1985)

13. Rudin W.: Functional analysis. New York: McGraw-Hill 1979

14. Weidmann J.: Linear operators in Hilbert spaces. New York Berlin Heidelberg: Springer 1980 